\title{
ON THE KREIN-RUTMAN THEOREM AND ITS APPLICATIONS TO CONTROLLABILITY
}

\author{
VU NGOC PHAT AND TRINH CONG DIEU \\ (Communicated by Palle E. T. Jorgensen)
}

\begin{abstract}
This paper extends Krein-Rutman's theorem on linear operators leaving an invariant cone in infinite-dimensional Banach spaces to multivalued convex functions. The result is applied to obtain necessary and sufficient conditions for global controllability and reachability of nonlinear discrete-time systems described by convex processes.
\end{abstract}

\section{INTRODUCTION}

The classical theorem of Krein-Rutman on linear operators leaving an invariant cone in Banach spaces obtained in [4] is as follows:

(K-R's theorem). Let $A \in L(X, X)$ be a linear bounded operator. Let $K$ be a cone with int $K \neq \varnothing$. If $A$ (int $K) \subset$ int $K$, then there is a nonzero functional $f^{*} \in K^{*}$ such that $A^{*} f^{*}=\lambda f^{*}, \lambda>0$.

This result has found a wide range of applications not only in linear operator theory but also in control theory ([3, 6-8] and others). Later, Korobov and Son [5] improved K-R's theorem in the following form: Let $A \in L(X, X)$ be a linear bounded operator. Let $K$ be a convex cone with int $K \neq \varnothing, K \neq X$. If $A K \subset K$, then there is a nonzero functional $f^{*} \in K^{*}$ such that $A^{*} f^{*}=\lambda f^{*}$, $\lambda \geq 0$.

Korobov and Son also successfully applied this theorem to derive necessary and sufficient conditions for controllability of linear systems with constrained controls in Banach spaces.

The purpose of this paper is to extend K-R's theorem for multivalued convex functions. This result will be applied to obtain necessary and sufficient conditions for controllability and reachability of nonlinear abstract systems described by discrete-time inclusion whose right-hand sides are convex processes. These systems include linear discrete-time systems with constrained controls and states in Banach spaces.

It is worthwhile to note that controllability results obtained in this paper can be considered as an extension to discrete-time inclusion of the corresponding controllability criteria of differential inclusion given in [2].

Received by the editors May 18, 1992.

1991 Mathematics Subject Classification. Primary 46A32, 47D20; Secondary 93B05. 


\section{Set-VAlued version of K-R's Theorem}

We first present some notation and definitions needed later. Let $X$ be an infinite-dimensional Banach space. $X^{*}$ denotes the dual topological space of $X$. The interior, the relative interior, the closure, the cone, and the linear subspace spanned by a set $M$ are denoted by int $M, \operatorname{ri} M, \operatorname{cl} M, \operatorname{con} M$, and sp $M$, respectively.

Let $M^{*}$ be the positive polar cone of a set $M$ defined by

$$
M^{*}=\left\{x^{*} \in X^{*}:\left\langle x^{*}, x\right\rangle \geq 0 \forall x \in M\right\} .
$$

To any multivalued function $F: X \rightarrow X$ we associate the domain, the image, and the graph of $F$ :

$$
\begin{gathered}
\operatorname{dom} F=\{x \in X: F(x) \neq \varnothing\}, \quad \operatorname{Im} F=\bigcup_{x \in X} F(x), \\
\operatorname{gr} F=\{(x, y): y \in F(x)\} .
\end{gathered}
$$

Definition 2.1. A multivalued function $F: X \rightarrow X$ is called convex, closed if gr $F$ is a convex, closed subset. If $\operatorname{gr} F$ is a convex, closed cone, the multivalued function $F$ is called a convex process, and it is strict if its domain is the whole space.

Definition 2.2. Let $F: X \rightarrow X$ be a multivalued function. We associate with $F$ the adjoint function $F^{*}$ defined by

$$
x^{*} \in F^{*}\left(y^{*}\right) \Leftrightarrow\left\langle y^{*}, y\right\rangle \geq\left\langle x^{*}, x\right\rangle \quad \forall(x, y) \in \operatorname{gr} F .
$$

Note that the adjoint function of a convex process is a natural extension of transposition of a linear operator. For example, if $A: X \rightarrow X$ is a bounded linear operator and $L$ is a convex, closed cone, then the multivalued function $F(x)=A x+L$ is a convex process, and we have

$$
F^{*}\left(x^{*}\right)= \begin{cases}A^{*} x^{*} & \text { if } x^{*} \in L^{*}, \\ \varnothing & \text { if } x^{*} \notin L^{*}\end{cases}
$$

As in [2], for every convex process $F: X \rightarrow X$, the adjoint function $F^{*}$ is upper semicontinuous (u.s.c) with convex weak compact values and $F(0)^{*}=$ $\operatorname{dom} F^{*}$.

Definition 2.3. If $\lambda x \in F(x), x \neq 0$, we shall say that $x \in \operatorname{dom} F$ is an eigenvector of $F$ and $\lambda$ is an eigenvalue of $F$.

Note that $F^{*}$ has an eigenvector if and only if $\operatorname{cl} \operatorname{Im}(F-\lambda I) \neq X$ for some $\lambda \in R$, where $I$ denotes the identity operator in $X$.

In the sequel, we denote by $F^{-1}$ the inverse function of $F$ defined by $F^{-1}(x)=\{y: x \in F(y)\}$.

Definition 2.4. Let $K$ be a cone. $K$ is invariant by $F$ iff $F(x) \subset K \forall x \in K$.

Throughout this paper we shall assume that the multivalued function $F: X \rightarrow$ $X$ is a strict convex process, and $0 \in F(0), k=1,2, \ldots$. 
Theorem 2.1. Let $K$ be convex cone, $K \neq X$, and int $K \neq \varnothing$. Assume that $K$ is invariant by $F$. Then $F^{*}$ has an eigenvector $x^{*} \in K^{*}$ with a nonnegative eigenvalue.

Proof. Since $F(0) \subset K, F(0)^{*} \supset K^{*}$. We have $F(0)^{*}=\operatorname{dom} F^{*}$. Then, for every $k^{*} \in K^{*}$, we obtain $F^{*}\left(k^{*}\right) \neq \varnothing$.

We shall prove that, for every $k^{*} \in K^{*}$,

$$
F^{*}\left(k^{*}\right) \cap K^{*} \neq \varnothing .
$$

Indeed, since $F(K) \subset K, K^{*} \subset F(K)^{*}$. By Corollary 22 [1, p. 144] we have

$$
F(K)^{*}=\left(F^{*}\right)^{-1}\left(K^{*}\right) \text {. }
$$

Therefore, for every $k^{*} \in K^{*}$, there is $x^{*} \in K^{*}$ such that $k^{*} \in\left(F^{*}\right)^{-1}\left(x^{*}\right)$. Then $x^{*} \in F^{*}\left(k^{*}\right)$, and hence

$$
x^{*} \in F^{*}\left(k^{*}\right) \cap K^{*} .
$$

Now let $x_{0} \in \operatorname{int} K$. We set $H=\left\{x^{*} \in K^{*}:\left\langle x^{*}, x_{0}\right\rangle=1\right\}$. It is easy to see that $H$ is a convex, weak compact subset in $X^{*}$. For every $x^{*} \in H$ we define the following multivalued function $G: H \rightarrow X^{*}$ by setting

$$
G\left(x^{*}\right)=\left\{y^{*}-\left\langle y^{*}, x_{0}\right\rangle x^{*}: y^{*} \in F\left(x^{*}\right) \cap K^{*}\right\} .
$$

We claim that:

(i) $G\left(x^{*}\right) \neq \varnothing$ for every $x^{*} \in H$,

(ii) $G(\cdot)$ is an upper semicontinuous multivalued function,

(iii) $G\left(x^{*}\right) \cap \operatorname{cl} \operatorname{con}\left(H-x^{*}\right) \neq \varnothing$ for every $x^{*} \in H$.

(i) and (ii) follow from (2.1) and from the above remark that $F^{*}\left(x^{*}\right)$ is a u.s.c. function. To prove (iii), for every $x^{*} \in H$, we take some $y^{*} \in K^{*}$ such that $y^{*} \in F^{*}\left(x^{*}\right) \cap K^{*}$. We have

$$
\bar{x}^{*}=y^{*}-\left\langle y^{*}, x_{0}\right\rangle x^{*} \in G\left(x^{*}\right) .
$$

If $y^{*}=0, \bar{x}^{*}=0 \in H-x^{*}$. If $y^{*} \neq 0$, we obtain

$$
\bar{x}^{*}=\left\langle y^{*}, x_{0}\right\rangle\left(\frac{y^{*}}{\left\langle y^{*}, x_{0}\right\rangle}-x^{*}\right) \in \operatorname{con}\left(H-x^{*}\right),
$$

because $\left\langle y^{*}, x_{0}\right\rangle>0$. Thus, we have

$$
\bar{x}^{*} \in G\left(x^{*}\right) \cap \operatorname{cl} \operatorname{con}\left(H-x^{*}\right),
$$

which implies (iii).

Now we can apply the theorem on the existence of zero of the multivalued function $[1$, p. 341$]$ to the function $G(\cdot)$. We obtain

$$
\exists x_{0}^{*} \in H: 0 \in G\left(x_{0}^{*}\right) .
$$

This implies that there is $y_{0}^{*} \in F^{*}\left(x_{0}^{*}\right) \cap K^{*}$ such that $y_{0}^{*}-\left\langle y_{0}^{*}, x_{0}^{*}\right\rangle x_{0}^{*}=0$, and hence $\left\langle y_{0}^{*}, x_{0}\right\rangle x_{0}^{*} \in F^{*}\left(x_{0}^{*}\right)$.

Therefore, we conclude that $F^{*}$ has the eigenvector $x_{0}^{*}$ in $K^{*}$ with the eigenvalue $\left\langle y_{0}^{*}, x_{0}\right\rangle \geq 0$, which completes the proof.

\section{ApPlications of CONTROLlability}

Let us consider the following discrete-time inclusion:

$$
x_{k+1} \in F\left(x_{k}\right), \quad k=0,1,2, \ldots,
$$


where the multivalued function $F: X \rightarrow X$ is assumed to be a strict convex process and $0 \in F(0)$.

It is known that the discrete-time inclusion (3.1) is a generalized discrete-time system to which the control systems with constrained controls

$$
x_{k}=f\left(x_{k}, u_{k}\right), \quad u_{k} \in \Omega,
$$

or control systems with feedbacks

$$
x_{k+1}=f\left(x_{k}, u_{k}\right), \quad u_{k} \in U\left(x_{k}\right),
$$

can be reduced by setting $F\left(x_{k}\right)=f\left(x_{k}, \Omega\right)$ or $F\left(x_{k}\right)=\left\{f(x, u): u \in U\left(x_{k}\right)\right\}$.

A point $x \in X$ is said to be null-controllable in $k$-time (reachable in $k$-time, resp.) if there is a solution $x_{0}, x_{2}, \ldots, x_{k}$, such that $x_{0}=x, x_{k}=0\left(x_{0}=0\right.$, $x_{k}=x$, resp.).

Let $\mathscr{R}_{k}$ and $\mathscr{C}_{k}$ be the sets of all reachable and null-controllable points in $k$-time of (3.1), respectively. Let

$$
\mathscr{R}=\bigcup_{k \geq 1} \mathscr{R}_{k}, \quad \mathscr{C}=\bigcup_{k \geq 1} \mathscr{C}_{k} .
$$

System (3.1) is said to be:

(i) globally null-controllable (globally reachable, resp.) in $k$-time if $\mathscr{C}_{k}=X$ $\left(\mathscr{R}_{k}=X\right.$, resp. $)$

(ii) globally null-controllable (globally reachable, resp.) if $\mathscr{C}=X \quad(\mathscr{R}=X$, resp.).

In the sequel, we shall say that $F$ satisfies the rank condition if int $F^{k}(0) \neq$ $\varnothing$, for some positive integer $k$ where

$$
F^{k}(0)=F(\underbrace{\ldots}_{k \text {-times }} F(0)) .
$$

Remark 3.1. As in [2], we also observe that the rank condition is equivalent to the following:

(i) $\exists k \geq 1$ such that $F^{k}(0)-F^{k}(0)=X$,

(ii) $\exists k \geq 1$ such that $F^{k}(0)^{*}=\{0\}$.

For example, if $F(x)=A x+L$, where $L$ is a convex closed cone and ri $L \neq \varnothing$, the rank condition is satisfied if and only if for some positive integer $N$

$$
\operatorname{sp}\left\{W, A W, \ldots, A^{N-1} W\right\}=X, \quad W=\operatorname{sp} L .
$$

This assertion can easily be proved by using the Banach open mapping theorem.

Theorem 3.1. Assume that $F$ satisfies the rank condition. Then the convex process (3.1) is globally reachable if and only if $F^{*}$ has no eigenvector with a nonnegative eigenvalue.

Proof. Necessity. Let process (3.1) be globally reachable. For every $\bar{x} \in \mathscr{R}$, there is a solution $\left(x_{0}, x_{1}, \ldots, x_{N}\right)$ of process (3.1) for some $N \geq 1$ such that $x_{0}=0, x_{N}=\bar{x}$. If $F^{*}$ has an eigenvector $x^{*}$ such that $\lambda x^{*} \in F^{*}\left(x^{*}\right), \lambda \geq 0$. By the definition of $F^{*}$, we have

$$
\left\langle x^{*}, \bar{x}\right\rangle \geq \lambda\left\langle x^{*}, x_{N-1}\right\rangle \geq \cdots \geq \lambda^{N}\left(x^{*}, 0\right)=0 .
$$


This, however, contradicts the assumption that $\mathscr{R}=X$.

Sufficiency. Assume to the contrary that process (3.1) is not globally reachable, i.e., $\mathscr{R} \neq X$. From the fact that

$$
\mathscr{R}_{N}=F^{N}(0),
$$

and from the rank condition, we have int $\mathscr{R} \neq \varnothing$. On the other hand, we note that $F(\mathscr{R}) \subset \mathscr{R}$, due to $0 \in F(0)$.

Therefore, by Theorem 2.1 there is $x^{*} \in X^{*}$ such that $\lambda x^{*} \in F^{*}\left(x^{*}\right), \lambda \geq 0$, which contradicts the assumption that $F^{*}$ has no eigenvector with a nonnegative eigenvalue.

Theorem 3.2. Assume that $F^{-1}$ satisfies the rank condition and $\operatorname{Im} F=X$. The convex process (3.1) is globally null-controllable if and only if $F^{*}$ has no eigenvector with a positive eigenvalue.

Proof. We first note that the convex process (3.1) is globally null-controllable if and only if the system

$$
x_{k+1} \in F^{-1}\left(x_{k}\right)
$$

is globally reachable. Since $F$ is a convex process and $\operatorname{Im} F=X, F^{-1}$ is also a convex process. Applying Theorem 3.1 to the multivalued function $F^{-1}$, we obtain that the process (3.2) is globally reachable if and only if $\left(F^{-1}\right)^{*}$ has no eigenvector with a nonnegative eigenvalue. On the other hand, from the fact [1, p. 139] that

$$
\left(F^{-1}\right)^{*}\left(x^{*}\right)=-\left(F^{*}\right)^{-1}\left(-x^{*}\right),
$$

it follows that $F^{*}$ has an eigenvector with an eigenvalue $\lambda>0$ if and only if $\left(F^{-1}\right)^{*}$ has an eigenvalue $\beta \geq 0$, which completes the proof.

In the case when $F(x)=A x+L$, where $A$ is a linear bounded operator and $L$ is a convex closed cone, ri $L \neq \varnothing$, we obtain the following immediate consequences of the above results:

Corollary 3.1. Let $F(x)=A x+L$. The convex process (3.1) is globally reachable if and only if

(i) $\operatorname{sp}\left\{W, A W, \ldots, A^{N-1} W\right\}=X$ for some $N \geq 1, W=\operatorname{sp} L$,

(ii) $A^{*}$ has no eigenvector $x^{*} \in L^{*}$ with a nonnegative eigenvalue.

Corollary 3.2. Let $F(x)=A x+L$. Assume that $\operatorname{Im} A=X$. The convex process (3.1) is globally null-controllable if and only if

(i) $\operatorname{sp}\left\{W, A W, \ldots, A^{N-1} W\right\}=X$ for some $N \geq 1$,

(ii) $A^{*}$ has no eigenvector $x^{*} \in L^{*}$ with a positive eigenvalue.

We obtain here the controllability results of $[6,8]$.

\section{ACKNOWLEDGMENTS}

The authors are grateful to the referee for some helpful remarks.

\section{REFERENCES}

1. J. P. Aubin and I. Ekeland, Applied nonlinear analysis, Wiley-Interscience, New York, 1984.

2. J. P. Aubin, H. Frankowska, and C. Olech, Controllability of convex processes, SIAM J. Control Optim. 24 (1986), 1192-1211. 
3. R. F. Curtain and A. J. Pritchard, Infinite dimensional linear systems theory, Lecture Notes in Control and Inform. Sci., vol. 8, Springer-Verlag, New York, 1978.

4. M. G. Krein and M. A. Rutman, Linear operator leaving invariant a cone in Banach spaces, Uspekhi Mat. Nauk 10 (1962), 199-325.

5. V. I. Korobov and N. K. Son, Controllability of linear systems in Banach spaces with restrictions on the control, Differentsial'nye Uravneniya 16 (1980), 1010-1022; English transl., Differential Equations 16, 633-642.

6. Nguyen Khoa Son, Controllability of linear discrete-time systems with constrained controls in Banach spaces, Control Cybernet. 1-2 (1981), 6-17.

7. Vu Ngoc Phat, Controllability of discrete-time dynamical systems, Ph.D. thesis, Azerbaidzan Univ., Bacu, USSR, 1984. (Russian)

8. __ Controllability of linear systems with multiple delays on controls and states in Banach spaces, Internat. J. Control 5 (1989), 1645-1654.

Department of Differential Equations, Institute of Mathematics, P. O. Box 631, Bo Ho, Hanoi, Vietnam

Department of Mathematics, Pedagogical Institute, Ho Chi Minh City, Vietnam 